\title{
PRODUÇÃO DE CONTEÚDO AUDIOVISUAL DE ENGENHARIA COMO MÉTODO DE BENEFICIAMENTO DO ENSINO APRENDIZAGEM NO CONTEXTO DA PANDEMIA DO COVID-19
}

DOI: 10.37702/2175-957X.COBENGE.2021.3370

JESSICA LAINE MENDES BERSAN - jessica.laine@engenharia.ufjf.br Universidade Federal de Juiz de Fora Rua Luiz Vicentin 151 36091-150 - Juiz de Fora - MG

Marcela Luz Coutinho - marcela.luz@engenharia.ufff.br Universidade Federal de Juiz de Fora Avenida Barão do Rio Branco 2376 36016-310 - Juiz de Fora - MG

Julia Righi de Almeida - julia.righi@engenharia.ufjf.br UFJF

Rua Monsenhor Gustavo Freire 21 36016-470 - Juiz de Fora - MG

Resumo: Entender as disciplinas de forma integrada e dinâmica é de extrema importância no processo de aprendizagem, principalmente quando se trata de cursos superiores de engenharia. $O$ ensino remoto emergencial (ERE) tornou-se uma ferramenta indispensável durante a pandemia do novo coronavírus, porém, este apresenta inúmeros impasses como dificuldade de concentração dos alunos devido à sobrecarga da grade curricular. No intuito de contribuir para a melhoria da aprendizagem dos alunos, o presente trabalho objetiva fazer uma discussão a respeito da importância e aplicabilidade do uso das redes sociais no ensino da engenharia. Além disso, utilizou-se como técnica a escala de Likert, com o intuito mensurar a avaliação dos discentes quanto a aspectos como aplicabilidade do método, grau de aprendizagem, qualidade do conteúdo, assimilação e grau de interesse. A pesquisa utilizada no presente artigo se classifica como exploratória, descritiva, aplicada, quantitativa e, quanto aos procedimentos técnicos, um estudo de caso. A pesquisa foi feita com estudantes que compõem as redes sociais de um grupo de alunos de uma universidade, no ano de 2021. Observou-se que o método criado é consistente e que os alunos apresentam grau de satisfação elevado com o 


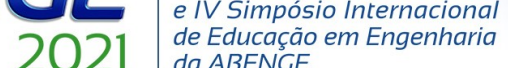 \\ 28 a 30 de SETEMBRO \\ "Formaçāo em Engenharia: Tecnologia, Inovaçâo e Sustentabilidade"}

projeto.

Palavras-chave: Educação. Pandemia. Engenharia. Redes sociais. Likert. 


\section{PRODUÇÃO DE CONTEÚDO AUDIOVISUAL DE ENGENHARIA COMO MÉTODO DE BENEFICIAMENTO DO ENSINO APRENDIZAGEM NO CONTEXTO DA PANDEMIA DO COVID-19}

\section{INTRODUÇÃO}

A pandemia do novo coronavírus, cientificamente identificado como SARS-COV-2, causador da doença COVID-19, apresentou vasto risco de contaminação, causando impactos inimagináveis à realidade humana nas mais diferentes proporções possíveis. Os efeitos negativos dessa pandemia se manifestam não apenas em um problema epidemiológico, com uma transmissão exponencial e um elevado número de mortes, mas também em outras consequências oriundas do isolamento social, feito com o intuito de conter a doença; e do fechamento de unidades escolares como creches, escolas e universidades e também empresas.

Segundo o monitoramento da situação das escolas no mundo, promovido pela UNESCO (2020), existem inúmeras consequências adversas do fechamento das instituições educativas, dentre as quais pode-se citar: a nutrição deficiente, pois muitas crianças e jovens dependem de refeições gratuitas ou com desconto fornecidas nas instituições; aumento das taxas de evasão, devido a necessidade de participação na geração de renda em famílias com condições financeiras desfavorecidas e maior exposição à violência e exploração, propiciados pelo isolamento social e pelo suporte dificultado fornecido pelas escolas e ONG's.

Em virtude de tal realidade, diversas organizações viram-se na necessidade de aderirem a novas práticas alternativas a fim de promover a continuidade dos processos de ensino-aprendizagem, feitos anteriormente de maneira presencial. Segundo Senhoras (2020), uma forma predominante para alavancar o contexto emergencial de estratégias de Ensino a Distância (EAD) é por meio do uso remoto das Tecnologias de Informação e Comunicação (TICs). Aliado às novas metodologias de ensino, o uso das redes sociais no ambiente formal de ensino estabelece um formato diferenciado de relacionamento entre professor-aluno dentro e fora da sala de aula. Isso se dá por meio do compartilhamento de informações de forma igualitária através do acesso ao mundo virtual, possibilitando tanto ao educando quanto ao professor fazer uso do conteúdo da rede para adquirir mais conhecimento (SILVA, VIEIRA, SCHINEIDER, 2010).

O objetivo desse artigo, portanto, é discutir a respeito da produção de materiais digitais disponibilizados nas mídias sociais pelo grupo no período de pandemia do novo coronavírus, sobre a importância dessa prática como forma de divulgação do ensino de engenharia e o impacto na conscientização e aprendizagem dos alunos acerca dos temas abordados. O estudo apresentará também uma discussão do contexto das redes sociais inseridas nas práticas de ensino remoto emergencial, uma análise da efetividade desses materiais e também, uma reflexão sobre essa metodologia de ensino, utilizando a escala de Likert para a mensuração dos dados. 


\section{AÇÕES DE ENSINO-APRENDIZAGEM REMOTOS MEDIADOS PELAS REDES SOCIAIS}

As redes possibilitam a comunicação, a interação e a colaboração, fatores relacionados às mais pertinentes teorias de aprendizagem, motivo pelo qual pesquisadores têm se interessado pelo tema, destacando a facilidade na formação de grupos entre estudantes e professores, na interação e compartilhamento de informações entre eles (PINTO et al., 2012). Além disso, elas podem ser um instrumento que corroboram positivamente para a manutenção do comprometimento educacional no curto prazo.

Segundo MATTAR (2013), a característica de leitura e produção de conteúdo cotidiano do aluno, como jogos, programas de TV e músicas, o processo de aprendizagem é facilitado contribuindo assim para a assimilação dos conceitos. Faz-se importante notar que o resultado do ensino remoto, utilizando as mídias sociais ou outros métodos de ensino a distância, dependem das condições infra estruturais e individuais de acessibilidade dos discentes, tais como acesso a computadores e internet de qualidade, bem como, grau de conhecimento digital dos docentes. Em virtude disso, foi visto em muitas instituições escolares a adoção de ações para apoio social e inclusão digital, como a Resolução $N^{\circ}$ 32.2020/05, que dentre outras finalidades, promoveu a manutenção de todas as bolsas e auxílios atualmente disponibilizadas às/aos estudantes de graduação e criação de auxílio de inclusão digital a fim de viabilizar o estudante na contratação de serviços que permitam o acesso à internet (MINISTÉRIO DA EDUCAÇÃO, 2005).

\section{MATERIAL E MÉTODOS}

A metodologia adotada nesta pesquisa se classifica, em relação aos seus objetivos, segundo Gil (2010), como exploratória e descritiva, uma vez que proporciona maior familiaridade com a problemática proposta e descreve as características de determinadas populações. A produção de material digital, incluindo vídeos, posts e quizzes interativos, foi feita pelo grupo de estudos, que engloba os cursos de Engenharia Ambiental e Sanitária e Engenharia Civil. Esse projeto atualmente conta com 16 alunos, entre voluntários e bolsistas e abrange Monitoria de Geologia (MO), Projetos de extensão (EX), Treinamento Profissional (TP) e Iniciação Científica (IC). As postagens eram feitas semanalmente e divididas por projetos, com o intuito de gerenciar as atividades e manter as redes sociais sempre ativas. Foram utilizados softwares de edição como Powtoon, Canva, Photoshop e Filmora. As redes sociais usadas como ferramentas de ensino e divulgação do trabalho foram Youtube, Blog, LinkedIn, Instagram e Facebook. Sempre se utilizaram imagens ilustrativas, posts interativos e vídeos chamativos com o intuito de facilitar a compreensão do público das redes sociais, que incluem alunos, professores e demais membros da sociedade.

Após o fim das postagens, foi elaborado um formulário de caráter exploratório qualitativo divulgado pelas redes sociais do grupo, com o intuito de mapear a percepção dos seguidores acerca dos materiais disponibilizados. A maioria das questões presentes no formulário exigiam respostas curtas e objetivas, utilizando-se da escala de Likert, que consiste de uma série de afirmações em que o respondente teve que optar pela escala que vai de "concordo totalmente" a "discordo totalmente", para cada afirmação. Foi utilizado também a inversão de parte das afirmações com o intuito de evitar o efeito de halo, ou seja, que o respondente marque uma alternativa em função unicamente da sua resposta para a afirmação anterior, o que poderia interferir de maneira negativa no mapeamento proposto. As exceções foram as perguntas sobre qual o curso de graduação e período, e a pergunta 
final, a qual o participante é livre para acrescentar alguma informação que considere relevante (NOGUEIRA, 2002).

\section{RESULTADOS E DISCUSSÃO}

\subsection{Análise quantitativa}

Foram feitas dez séries de conteúdos disponibilizadas nas redes sociais do grupo. Os projetos envolvidos foram Monitoria de Geologia (MO), Treinamento Profissional (TP), Extensão (EX) e Iniciação Científica (IC). As postagens eram feitas semanalmente e seu período de duração foi de março de 2020 a março de 2021. O quadro-resumo com os principais tópicos citados encontra-se na Tabela 1.

Tabela 1 - Quadro resumo das séries.

\begin{tabular}{c|c|c|c}
\hline Nome Série & $\begin{array}{c}\text { Formato do } \\
\text { conteúdo }\end{array}$ & $\begin{array}{c}\text { Número de } \\
\text { Postagens }\end{array}$ & $\begin{array}{c}\text { Projetos } \\
\text { relacionados }\end{array}$ \\
\hline Compostagem & Vídeos & 7 & $\mathrm{TP}$ \\
\hline Diferenças entre aterros sanitários, controlados e lixões & Vídeos & 5 & $\mathrm{TP}$ \\
\hline Geoquiz & Stories & 13 & $\mathrm{MO}$ \\
\hline Compostagem do Zero & Vídeos & 21 & $\mathrm{TP}$ \\
\hline Aterroflix & Vídeos & 4 & $\mathrm{TP}$ \\
\hline Transporte de contaminantes & Vídeos & 3 & $\mathrm{IC}$ \\
\hline Geologia nas Escolas & Vídeos & 7 & $\mathrm{EX}$ \\
\hline Conhecimentos em extensão & Vídeos & 6 & $\mathrm{EX}$ \\
\hline Análise de estabilidade & Vídeos & 5 & $\mathrm{IC}, \mathrm{TP}$ \\
\hline Artigos e TCC & Posts & 30 & $\mathrm{TP}$ \\
\hline
\end{tabular}

Fonte: Os autores.

Utilizou-se da produção de conteúdo audiovisual como uma forma de continuação das atividades que ficaram suspensas em virtude do surgimento do novo coronavírus. A crescente utilização de ferramentas de educação a distância fez-se necessária neste momento histórico de amplitude mundial. Nesse sentido, a divulgação de materiais nas redes sociais apresentou-se como uma ferramenta interessante de continuidade dos projetos. O quadro resumo com as informações citadas pode ser consultado na Tabela 2.

Tabela 2 - Quadro resumo com o conteúdo abordado.

\begin{tabular}{c|l}
\hline Nome Série & \multicolumn{1}{c}{ Conteúdo abordado } \\
\hline Compostagem & $\begin{array}{l}\text { Temas que permeiam o processo tais como mitos e verdades sobre } \\
\text { compostagem, como construir uma composteira doméstica, o que é } \\
\text { compostagem e acerca do dia Mundial do Meio Ambiente. O objetivo dessa } \\
\text { série era desmistificar e ensinar os conceitos iniciais do processo. }\end{array}$ \\
\hline Diferenças entre & $\begin{array}{l}\text { Nessa série foi feita inicialmente uma contextualização acerca da problemática } \\
\text { dos resíduos sólidos urbanos (RSU) no Brasil, abordando tópicos como } \\
\text { constituição do lixo dos municípios brasileiros, excesso de embalagens, } \\
\text { controlados e lixões } \\
\text { consumo excessivo e descarte inadequado. Posteriormente, foi feita a } \\
\text { diferenciação entre lixão, aterro controlado e aterro sanitário. }\end{array}$
\end{tabular}


Geoquiz

Compostagem do Zero

Aterroflix

Transporte de contaminantes

Geologia nas Escolas

Conhecimentos em extensão

Análise de estabilidade
Foram feitas postagens interativas semanais nos stories do Instagram com 0 objetivo de ensinar os principais tópicos da disciplina de Geologia Ambiental e Elementos de Geologia, ambas constituintes da grade curricular obrigatória dos cursos de Engenharia Ambiental e Sanitária e Engenharia Civil da universidade. As publicações eram feitas por meio de imagens, vídeos e questões de múltipla escolha onde o aluno deveria marcar a opção correta, seguida de uma resposta curta e de fácil compreensão. O quiz abarcou os principais tópicos da grade curricular tais como minerais, rochas e solos formados e buscou de uma forma lúdica e interativa, contribuir para a compreensão da disciplina.

Fez-se uma continuação da temática iniciada na série sobre compostagem, abarcando os mais diversos temas relacionados à prática tais como panorama dos RSU no Brasil, Política Nacional de Resíduos Sólidos, aterro sanitário, tipos de resíduos, resíduo orgânico, impacto do lixo orgânico nos aterros e lixões, por que devemos compostar, tipos de compostagem, o que pode e o que não pode ser colocado na composteira, etapas e subprodutos da compostagem, o que fazer com os subprodutos, aplicações da compostagem e dia a dia de uma composteira doméstica.

A série buscou unir de uma maneira inusitada a logo de uma provedora global de filmes e séries de televisão com a temática dos aterros sanitários, com o objetivo de chamar a atenção dos estudantes com temas abordados em duas disciplinas eletivas dos cursos de engenharia já lecionados na universidade. Foram abordados tópicos como panorama aterros no Brasil, legislação, fases da implantação dos aterros, triagem dos resíduos, impermeabilização, sistemas de drenagens interna e superficial, geossintéticos, instrumentação e topografia, operação, cobertura diária e compactação dos resíduos, monitoramento geotécnico e uso de drones.

A série "Transporte de Contaminantes" explicou o projeto de iniciação científica (IC). Foi abordado sobre as fontes de contaminação, importância do estudo do transporte de contaminantes, método de ocorrência e demonstração da construção de um equipamento para ensaio em coluna no laboratório da universidade, feito com o intuito de simular o transporte de contaminante no solo.

A série objetiva dar prosseguimento ao projeto de extensão (EX) e levar as atividades que seriam feitas presencialmente de maneira remota para a população, em especial para os alunos do ensino médio, que são o foco do projeto. O intuito é fazer com que toda a população se interesse por essa área da Engenharia ainda pouco explorada e que carece de profissionais para o seu pleno desenvolvimento. Foram abordados tópicos acerca da Geologia e Geotecnia ambiental como minerais, rochas, solos e resíduos.

A série "Conhecimentos em Extensão" tem o objetivo de contextualizar sobre o histórico dos projetos de extensão nas universidades, seus impactos e sua importância. Além disso, a série mostrou também a necessidade de ampliação desse tipo de projetos nos cursos de engenharia, como forma de retribuição à sociedade e manutenção do papel cidadão dos futuros profissionais do meio. A série objetiva apresentar o software SLOPE/W, pertencente ao pacote GeoStudio da Geoslope, como uma ferramenta de análise da estabilidade de taludes de aterros sanitários. Os materiais apresentaram a importância do programa na engenharia, quais são suas aplicações e também um tutorial de como manusear o programa. Foi ensinado como desenhar um aterro sanitário, como calcular seu fator de segurança e também como analisá-lo, baseandose na legislação vigente.

Artigos e TCC $\quad$ A série contou com a publicação de todos os artigos científicos feitos pelos membros do grupo, e também pelos Trabalhos de Conclusão de Curso (TCCs) orientados pela professora orientadora. Os documentos eram divulgados semanalmente no Linkedln, disponibilizados para download e podiam ser consultados livremente. 
Ao final do conteúdo, foi elaborado um formulário dividido em duas frentes. A primeira relacionada a dados pessoais dos respondentes e a segunda relacionada à percepção dos mesmos relacionado aos materiais confeccionados pelo grupo. Este recebeu respostas por quinze dias e foi divulgado nas redes sociais do grupo.

\subsection{Análise qualitativa}

O formulário contou com a participação de 118 respondentes, o que corresponde em média a $20 \%$ do espaço amostral, totalizado pelo número de seguidores somados de todas as redes sociais do grupo. Em relação às perguntas gerais que iniciaram o formulário, foram feitas duas considerações principais para se entender o perfil da amostra estudada, as quais podem ser encontradas nas Figuras 1 e 2.

Figura 1 - Constituição da amostra do estudo.

Constituição da amostra do estudo (\%)

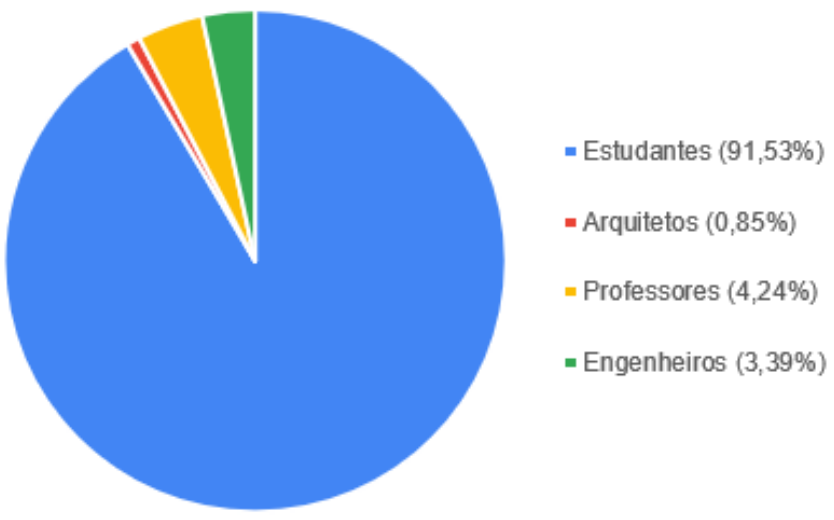

Fonte: Os autores.

Figura 2 - Constituição dos cursos de graduação dos estudantes da amostra do estudo.

Cursos de graduação dos estudantes respondentes (\%)
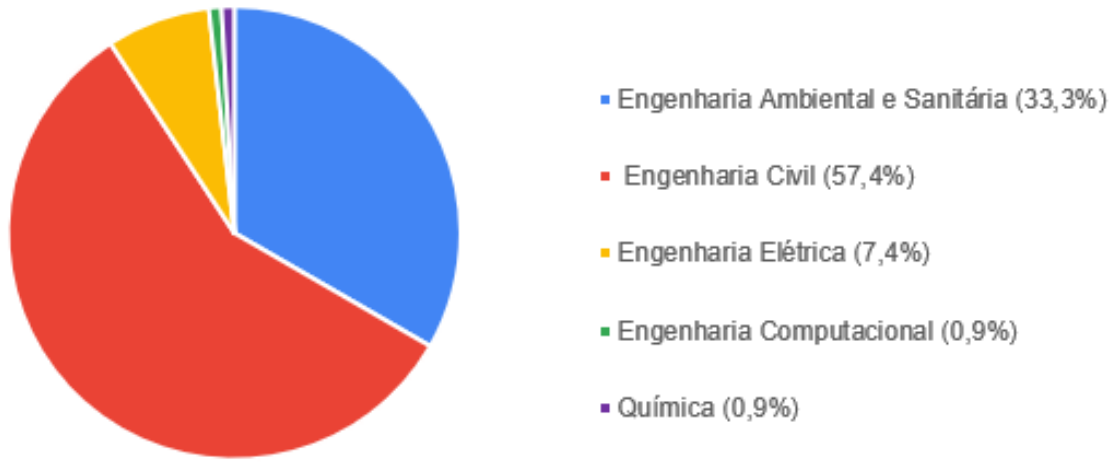

Fonte: Os autores. 
Em relação à frente qualitativa, as perguntas deveriam ser respondidas em uma escala de 1 a 5 , conforme mostra a Tabela 3.

Tabela 1 - Quadro resumo das séries.

\begin{tabular}{c|c|c|c|c|c|}
\hline \multirow{2}{*}{} & \multicolumn{5}{|c|}{ Nível de concordância } \\
\cline { 2 - 6 } & $\begin{array}{c}\text { Discordo } \\
\text { Totalmente }\end{array}$ & $\begin{array}{c}\text { Discordo } \\
\text { parcialmente }\end{array}$ & Indiferente & $\begin{array}{c}\text { Concordo } \\
\text { parcialmente }\end{array}$ & $\begin{array}{c}\text { Concordo } \\
\text { totalmente }\end{array}$ \\
\hline Pesos & 1 & 2 & 3 & 4 & 5 \\
\hline
\end{tabular}

Fonte: Os autores.

Foram feitas 10 perguntas a respeito do conteúdo disponibilizado nas redes sociais do grupo. É importante ressaltar que foi utilizado também a inversão de parte das afirmações com o intuito de evitar o efeito de halo, ou seja, que o respondente marque uma alternativa em função unicamente da sua resposta para a afirmação anterior. Estas podem ser podem ser consultadas na Tabela 4.

Tabela 4 - Descrição das perguntas contidas no formulário de avaliação.

\begin{tabular}{|c|c|}
\hline Nomeação & Perguntas \\
\hline 1 & $\begin{array}{l}\text { Considero que a metodologia empregada no EAD da engenharia poderia } \\
\text { utilizar de outras ferramentas como séries de vídeos nas redes sociais. }\end{array}$ \\
\hline II & $\begin{array}{l}\text { As formas de ensino expositivas adotadas na engenharia atualmente são } \\
\text { suficientes e eficazes na minha opinião. }\end{array}$ \\
\hline III & Considero que a metodologia empregada no EAD da engenharia é adequada. \\
\hline IV & $\begin{array}{l}\text { Acredito que a disponibilização dos conteúdos em formato digital facilita a } \\
\text { aprendizagem dos alunos. }\end{array}$ \\
\hline V & $\begin{array}{l}\text { Creio que é possível aprender e assimilar conteúdos disponíveis nas redes } \\
\text { sociais, mesmo que essas não tenham essa principal finalidade. }\end{array}$ \\
\hline $\mathrm{VI}$ & $\begin{array}{l}\text { Sigo perfis de educação no Instagram, Youtube e Linkedln e acredito que esta } \\
\text { é uma forma crescente e eficiente de aprendizagem. }\end{array}$ \\
\hline VII & $\begin{array}{l}\text { A adoção de novas técnicas de ensino como memória fotográfica e quizzes } \\
\text { facilitam o entendimento dos temas da graduação. }\end{array}$ \\
\hline VIII & $\begin{array}{l}\text { Creio que a confecção de materiais didáticos em diferentes formatos como } \\
\text { vídeos e posts são uma ferramenta muito útil de aprendizagem. }\end{array}$ \\
\hline IX & $\begin{array}{l}\text { Acredito que os temas das séries criadas nas redes sociais do grupo } \\
\text { contribuíram para a minha formação acadêmica. }\end{array}$ \\
\hline $\mathrm{X}$ & $\begin{array}{l}\text { As séries criadas possuem relação direta com algumas disciplinas ofertadas } \\
\text { no meu curso de graduação. }\end{array}$ \\
\hline
\end{tabular}

Fonte: Os autores.

Após a coleta dos resultados, estes foram tabulados com o auxílio do software Excel. Fez-se o valor da concordância multiplicado pelo número de respostas recebidas e por fim, a média das concordâncias, conforme a Tabela 5.

Tabela 5 - Valor por ordem de concordância das variáveis.

\begin{tabular}{c|c|c|c|c|c|c}
\hline Perguntas & $\begin{array}{c}\text { Discordo } \\
\text { Totalmente }\end{array}$ & $\begin{array}{c}\text { Discordo } \\
\text { parcialmente }\end{array}$ & Indiferente & $\begin{array}{c}\text { Concordo } \\
\text { parcialmente }\end{array}$ & $\begin{array}{c}\text { Concordo } \\
\text { totalmente }\end{array}$ & Média \\
\hline I & 10 & 88 & 27 & 204 & 20 & 2,96 \\
\hline II & 20 & 104 & 24 & 136 & 20 & 2,58 \\
\hline III & 2 & 16 & 42 & 176 & 250 & 4,12 \\
\hline IV & 0 & 4 & 3 & 168 & 365 & 4,58 \\
\hline
\end{tabular}


28 a 30 de SETEMBRO

\begin{tabular}{c|c|c|c|c|c|c}
\hline $\mathrm{V}$ & 2 & 8 & 21 & 188 & 290 & 4,31 \\
\hline $\mathrm{VI}$ & 2 & 2 & 21 & 152 & 350 & 4,47 \\
\hline $\mathrm{VII}$ & 0 & 6 & 15 & 120 & 400 & 4,58 \\
\hline $\mathrm{VIII}$ & 0 & 0 & 9 & 128 & 415 & 4,68 \\
\hline $\mathrm{IX}$ & 1 & 4 & 60 & 116 & 330 & 4,33 \\
\hline $\mathrm{X}$ & 0 & 4 & 60 & 92 & 365 & 4,42 \\
\hline
\end{tabular}

Fonte: Os autores.

A escala Likert verifica o grau de concordância ou não das afirmativas, obtendo- se os resultados através da pontuação atribuída a cada resposta. Com base na escala proposta de 5 pontos, as afirmativas com valor de média menor que 3 são consideradas discordantes e devem ser trabalhadas, e as afirmativas com média maior que 3 são consideradas concordantes e devem manter uma manutenção constante (LUDWIG et al., 2015). A Figura 3 apresenta de forma gráfica o desempenho de cada variável aplicada no decorrer de 12 meses de postagem de conteúdo.

Figura 3 - Análise gráfica das médias.

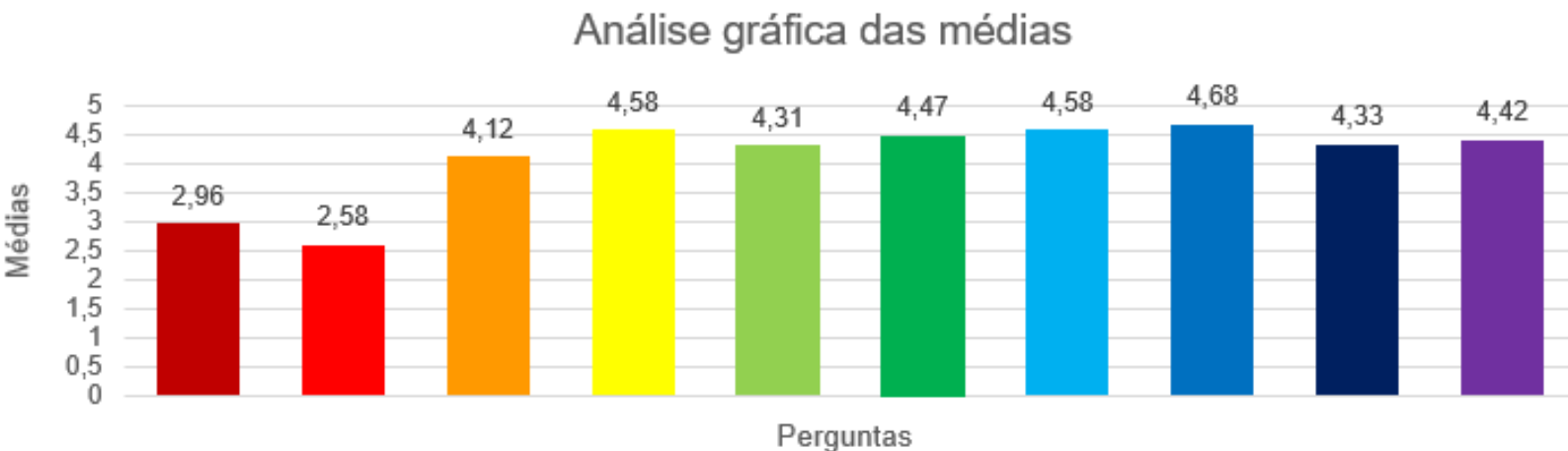

- Considero que a metodologia empregada no EAD da engenharia é adequada

- As formas de ensino expositivas adotadas na engenharia atualmente são suficientes e eficazes na minha opinião

- Considero que a metodologia empregada no EAD da engenharia poderia utilizar de outras ferramentas como séries de vídeos nas redes sociais Acredito que a disponibilização dos conteúdos em formato digital facilita a aprendizagem dos alunos

- Creio que é possivel aprender e assimilar conteúdos disponíveis nas redes sociais, mesmo que essas não tenham essa principal finalidade - Sigo perfis de educação no Instagram, Youtube e Linkedin e acredito que esta é uma forma crescente e eficiente de aprendizagem

- A adoção de novas técricas de ensino como memória fotográfica e quizzes facilitam o entendimento dos temas da graduação

- Creio que a confecção de materiais didáticos em diferentes formatos como vídeos e posts são uma ferramenta muito útil de aprendizagem

- Acredito que os temas das séries criadas nas redes sociais do Geoportal contribuíram para a minha formação acadêmica

- As séries criadas possuem relação direta com algumas disciplinas ofertadas no meu curso de graduação

Fonte: Os autores.

Conforme mostra a Tabela 5, obteve-se um resultado satisfatório na maioria das afirmações, já que elas obtiveram média acima de 3, e a média geral apresentou o valor de 4,10 . Observa-se que apenas as duas primeiras afirmações obtiveram médias inferiores às globais.

A afirmativa (I) referente a adequação do Ensino a Distância (EAD) no contexto da engenharia, indica que esta metodologia deve ser revista e apresenta falhas na percepção dos alunos. Tal valor pode ser explicado também pelo fato de que a metodologia de ensino empregada nas universidades brasileiras no contexto da pandemia não foi o EAD, mas sim o ERE.

De acordo com o portal "Desafios da Educação" do Grupo A, maior empresa de tecnologia para a educação do Brasil, o ERE é uma medida extraordinária e temporária aprovada pelo Ministério da Educação (MEC), para que as instituições de ensino possam cumprir o cronograma das aulas presenciais em situações anormais, como o caso da pandemia do COVID-19. Dentre as características principais do ERE é interessante citar a virtualização do ambiente de aprendizagem, que normalmente apresenta-se improvisado e 
inadequado, e a diversificação das metodologias de avaliação, que podem causar sobrecarga nos discentes. Em contrapartida, ainda segundo o portal, o EAD é um modelo de ensino remoto feito de forma planejada, e não emergencial. Todo ou parte do curso é ministrado a distância e apresenta ferramentas complementares como apoio de tutores, recursos de vídeo, e-books, podcasts, entre outros. Suas características são baseadas na flexibilidade e seus pontos principais são investimentos em ambientes virtuais de aprendizagem, como Ambiente Virtual de Aprendizagem (AVA) e metodologias de suporte ao aluno.

A afirmativa referente ao método de ensino expositivo na engenharia (II), também merece especial atenção. Percebe-se uma necessidade dos alunos em mudanças na metodologia empregada. O método expositivo, que pode ser feito por meio de apresentações em Power Point por exemplo, permite que uma maior quantidade e profundidade de conteúdos sejam ministrados, entretanto, apresenta uma menor participação dos alunos. Esta parece a melhor condição para balancear a abrangência e complexidade de conteúdo exigido pelas grades curriculares, "assumindo-se que toda transmissão de informações resulta necessariamente em aprendizagem, o que não é sempre verdadeiro"(RIBEIRO, 2008).

Em relação às demais afirmações, percebe-se a indispensabilidade de adequação do sistema existente atualmente com as necessidades do ambiente de ensino remoto. A criação de materiais em mídias alternativas como em redes sociais apresenta especial destaque e os conteúdos criados pelo grupo e divulgados em suas redes sociais, evidenciaram elevada contribuição para a formação dos alunos dos mais diversos cursos de engenharia, tais como civil, ambiental e sanitária, elétrica e computacional.

\section{CONCLUSÃO}

A persistência do modelo convencional no ensino de engenharia adotado pelas instituições de ensino superior (IES) apresenta inúmeros impasses, dentre os quais podese citar a longevidade das universidades, que apresentam processos extremamente burocráticos e conservacionistas, a inexperiência com ensino dos docentes e a insuficiência de conceitos chaves dos discentes.

Em relação aos docentes, percebe-se uma dificuldade em motivar os alunos a aprenderem matérias básicas, o que prejudica o entendimento posterior das teorias mais avançadas. Somado a isso, é interessante citar um certo comodismo que impede a adoção de novas metodologias de ensino além de ineficiência de didática em sala de aula. Não obstante, muitos autores concordam ser mais vantajoso ensinar o aluno a aprender do que arriscar transmitir-Ihe todos os conceitos e esperar que ele os incorpore à prática no futuro (RIBEIRO, 2008).

Acerca dos discentes, percebe-se uma deficiência de bagagem de conceitos básicos fundamentais para o ensino superior. Somado a isso, o uso excessivo de tecnologia causa um imediatismo na busca por conhecimento que não condiz com o método expositivo normalmente adotado pelos docentes.

As Diretrizes Curriculares Nacionais dos Cursos de Engenharia (DCN) indicam uma direção dos cursos de graduação com estruturas mais flexíveis, permitindo que o futuro profissional a ser formado tenha, dentre outras competências, ênfase na síntese e na transdisciplinaridade, preservação do meio ambiente e forte vinculação entre teoria e prática.

Em consequência disso, conclui-se que o emprego de mídias digitais no ensino da engenharia, apresenta-se como um novo método de aprendizagem com elevada adesão pelos discentes. Dentre suas vantagens, pode-se citar a melhoria da aprendizagem e 
aumento do interesse pelo conteúdo, propiciado pela utilização de ferramentas que chamem mais a atenção dos alunos, como conteúdos de menor duração, interação e ilustrações que facilitem o entendimento.

Faz-se importante citar que é crescente o número de perfis de projetos, alunos e professores que utilizam deste método de disseminação de conhecimento. Assim sendo, a adesão de modelos de aprendizagem virtuais que incorporem os processos já existentes, apresenta-se como uma maneira inovadora de ensino capaz de promover ambientes de aprendizagem colaborativos, críticos e construtivistas.

\section{REFERÊNCIAS}

UNESCO - United Nations Educational, Scientific and Cultural Organization. "COVID-19 Educational Disruption and Response". UNESCO Website [06/05/2020]. Disponível em: $<$ https://en.unesco.org/covid19/educationresponse/consequences>.

Acesso

em 03/12/2020.

MATTAR, J. Web 2.0 e redes sociais na educação. São Paulo: Artesanato Educacional, 2013.

MINISTÉRIO DA EDUCAÇÃO. RESOLUÇÃO № 32.2020, DE 05 DE AGOSTO DE 2020. Estabelece normas para desenvolvimento de ações de apoio social e inclusão digital na UFJF no contexto de suspensão das atividades presenciais em decorrência da pandemia COVID-19. Disponível em: <https://www2.ufjf.br/consu/wp content/uploads/sites/33/2020/08/Resolu\%C3\%A7\%C3\%A3o-32.2020_Assinada.pdf>. Acesso em 03/12/2020.

NOGUEIRA, R. Elaboração e análise de questionários: Uma revisão da literatura dos conceitos a um caso real. Rio de Janeiro: UFRJ/COPPEAD, 26p. 2002. LUDWIG, J. P.; FAIZ, E. B.; PALOSCHI, R. B.; SOUZA, J. Planejamento estratégico: análise de eficácia da metodologia aplicada por meio da Escala Likert. Disponível em: <http://www.revistaespacios.com/a15v36n16/15361609.html>. Acesso em 10/02/2001.

PINTO, S. C. C. S. et al. Redes Sociais: impactos, desafios e pesquisas no cenário educacional. In: SCHNEIDER, H. N.; LACKS, S. (org.). Educação no século XXI: desafios e perspectivas. São Cristóvão: Editora UFS, 2012.

RIBEIRO, L. R. de C. Aprendizagem baseada em problemas (PBL) na educação em engenharia. Revista de Ensino de Engenharia, v. 27, n. 2, p. 23-32, 2008.

SCHNEIDER, S. O. Comparação entre aula expositiva e aprendizagem baseada em problemas no desenvolvimento de conceitos e competências na engenharia. Trabalho de Conclusão de Curso de Engenharia Civil. Porto Alegre, 2019. Disponível em: https://www.lume.ufrgs.br/bitstream/handle/10183/206049/001112301.pdf?sequence=1 \&isAllowed=y.

SENHORAS, Elói Martins. CORONAVÍRUS E EDUCAÇÃO: análise dos impactos assimétricos. Boletim de conjuntura. Boa Vista, v. 2, n.2, p. 128-136, 2020.

SILVA, A. L.; VIEIRA, E. S.; SCHNEIDER, H. N. O uso das redes sociais como método alternativo de ensino para jovens: análise de três projetos envolvendo comunidades virtuais. In: Colóquio Internacional Educação e Contemporaneidade, 4., 2010, São 
Cristóvão. Anais do IV Colóquio Internacional Educação e Contemporaneidade. São

Cristóvão: EDUCON, 2010.

\title{
PRODUCTION OF AUDIOVISUAL ENGINEERING CONTENT AS A METHOD OF IMPROVEMENT OF TEACHING-LEARNING IN THE CONTEXT OF THE PANDEMIC COVID-19 - COBENGE 2021
}

\begin{abstract}
Understanding the disciplines in an integrated and dynamic way is extremely important in the learning process, especially when it comes to higher engineering courses. Emergency remote education (ERE) became an indispensable tool during the pandemic of the new coronavirus, however, it presents numerous impasses as a difficulty in concentrating students due to the overload of the curriculum. In order to contribute to the improvement of students' learning, this paper aims to discuss the importance and applicability of the use of social networks in engineering education. In addition, the Likert scale was used as a technique, in order to measure the evaluation of students regarding aspects such as the applicability of the method, degree of learning, quality of content, assimilation and degree of interest. The research used in this article is classified as exploratory, descriptive, applied, quantitative and, as for technical procedures, a case study. The research was carried out with students who make up the social networks of a group of students from a university, in the year 2021. It was observed that the method created is consistent and that the students have a high degree of satisfaction with the project.
\end{abstract}

Keywords: Education, Pandemic, Engineering, Social networks, Likert. 